\title{
Inactivation of the tight junction gene CLDN11 by aberrant hypermethylation modulates tubulins polymerization and promotes cell migration in nasopharyngeal carcinoma
}

Hsin-Pai Li $i^{1,2,5,8^{*}+}$, Chen-Ching Peng ${ }^{1,2,5+}$, Chih-Ching Wu ${ }^{3,5,7}$, Chien-Hsun Chen ${ }^{1,2}$, Meng-Jhe Shih', ${ }^{1,2}$ Mei-Yuan Huang ${ }^{2}$, Yi-Ru Lai ${ }^{2}$, Yung-Li Chen ${ }^{4}$, Ting-Wen Chen ${ }^{5,6}$, Petrus Tang ${ }^{1,5,6}$, Yu-Sun Chang 1,5,7, Kai-Ping Chang ${ }^{7}$ and Cheng-Lung $\mathrm{Hsu}^{8}$

\begin{abstract}
Background: Aberrant hypermethylation of cellular genes is a common phenomenon to inactivate genes and promote tumorigenesis in nasopharyngeal carcinoma (NPC).

Methods: Methyl binding domain (MBD)-ChIP sequencing of NPC cells, microarray data of NPC biopsies and gene ontology analysis were conducted to identify a potential tumor suppressor gene CLDN11 that was both hypermethylated and downregulated in NPC. Bisulfite sequencing, qRT-PCR, immunohistochemistry staining of the NPC clinical samples and addition of methylation inhibitor, 5'azacytidine, in NPC cells were performed to verify the correlation between DNA hypermethylation and expression of CLDN11. Promoter reporter and EMSA assays were used to dissect the DNA region responsible for transcription activator binding and to confirm whether DNA methylation could affect activator's binding, respectively. CLDN11 was transiently overexpressed in NPC cells followed by cell proliferation, migration, invasion assays to characterize its biological roles. Co-immunoprecipitation experiments and proteomic approach were carried out to identify novel interacting protein(s) and the binding domain of CLDN11. Anti-tumor activity of the CLDN11 was elucidated by in vitro functional assay.

(Continued on next page)
\end{abstract}

\footnotetext{
*Correspondence: paili@mail.cgu.edu.tw

${ }^{\dagger}$ Equal contributors

'Graduate Institute of Biomedical Sciences, Chang Gung University, No.259,

Wenhua 1st Rd., Guishan Dist., Taoyuan City 333, Taiwan

${ }^{2}$ Department of Microbiology and Immunology, Chang Gung University,

No.259, Wenhua 1st Rd., Guishan Dist., Taoyuan City 333, Taiwan

Full list of author information is available at the end of the article
}

(c) The Author(s). 2018 Open Access This article is distributed under the terms of the Creative Commons Attribution 4.0 International License (http://creativecommons.org/licenses/by/4.0/), which permits unrestricted use, distribution, and reproduction in any medium, provided you give appropriate credit to the original author(s) and the source, provide a link to the Creative Commons license, and indicate if changes were made. The Creative Commons Public Domain Dedication waiver (http://creativecommons.org/publicdomain/zero/1.0/) applies to the data made available in this article, unless otherwise stated. 
(Continued from previous page)

Results: A tight junction gene, CLDN11, was identified as differentially hypermethylated gene in NPC. High methylation percentage of CLDN11 promoter in paired NPC clinical samples was correlated with low mRNA expression level. Immunohistochemistry staining of NPC paired samples tissue array demonstrated that CLDN11 protein expression was relatively low in NPC tumors. Transcription activator GATA1 bound to CLDN11 promoter region -62 to -53 and its DNA binding activity was inhibited by DNA methylation. Re-expression of CLDN11 reduced cell migration and invasion abilities in NPC cells. By co-immunoprecipitation and liquid chromatographytandem mass spectrometry LC-MS/MS, tubulin alpha-1b (TUBA1B) and beta-3 (TUBB3), were identified as the novel CLDN11-interacting proteins. CLDN11 interacted with these two tubulins through its intracellular loop and Cterminus. Furthermore, these domains were required for CLDN11-mediated cell migration inhibition. Treatment with a tubulin polymerization inhibitor, nocodazole, blocked NPC cell migration.

Conclusions: CLDN11 is a hypermethylated and downregulated gene in NPC. Through interacting with microtubules TUBA1B and TUBB3, CLDN11 blocks the polymerization of tubulins and cell migration activity. Thus, CLDN11 functions as a potential tumor suppressor gene and silencing of CLDN11 by DNA hypermethylation promotes NPC progression.

Keywords: Nasopharyngeal carcinoma, Methylation, Tight junction, CLDN11

\section{Background}

Aberrant DNA hypermethylation of tumor suppressor genes is a well-studied epigenetic gene silencing event, which promotes cancer formation and progression [1-3]. Nasopharyngeal carcinoma (NPC) is an Epstein-Barr virus (EBV)-associated head and neck cancer. The oncogenic viral latent membrane protein 1 (LMP1) can activate host DNA methyltransferase 1 (DNMT1), causing aberrant hypermethylation, transcription inactivation, and loss of function of tumor suppressor genes in NPC [4-8]. We previously demonstrated that HoxA2 (coding gene) [9] and miR-148a (noncoding gene) [10] are the two tumor suppressor genes silenced through aberrant DNA hypermethylation in NPC tumors.

To characterize genome-wide, aberrantly hypermethylated and downregulated genes in NPC cells, we employed next generation sequencing (NGS) approach to identify the NPC-specific methylated genes. First, we enriched the methylated genomic DNA from NPC cell line C666.1 (sample) and immortalized normal NP cell line NP69 (control), respectively, by using methylated DNA affinity column containing methyl binding protein, MBD (Methylminer Methylated DNA Enrichment Kit). We then collected and PCR-amplified the methylated DNA from the methylated DNA affinity column. The amplified DNA was subjected to high-throughput NGS sequencer (Illumina GAII; Genomic Center Yang Ming University, Taiwan). Sequencing data were further analyzed by DNAnexus ChIP-seq algorithm. Significantly enriched and differentially methylated genes (experimental versus background 21.5) in C666.1 when compared with that of the NP69 were identified (Fig. 1). These NPC-specific methylated genes are potential tumor suppressor genes which could be epigenetically inactivated during NPC tumorigenesis. Second, we intersected the above NPC hypermethylated gene list (NGS) with the downregulated genes selected from our in-house NPC tumors cDNA microarray (Affymetrix HG U133 plus 2.0 chip) to correlate the notion that hyperemethylated genes are often transcriptionally repressed. The cDNA expression profiling was compared between nine NPC tumor samples and one pooled adjacent normal tissues [11]. Gene expression $>1$.3-fold reduction in NPC tumor tissues when compared with that of pooled non-tumors were selected. After intersecting the two gene lists, we identified a differentially methylated and downregulated gene candidate claudin 11 (CLDN11) in NPC cells. Claudins are a family of genes with 27 members. They are integral membrane proteins containing four transmembrane domains which serve as crucial tight junction components and cell barrier for cells [12-17]. CLDN11 is hypermethylated and silenced in bladder cancer [18], gastric cancer [19], oral leukoplakias [20] and malignant melanoma [21]. The reduction in CLDN11 expression is associated with increase in invasiveness in multiple cancer types [18, 22, 23]; the reintroduction of this gene reverses the cancerous phenotype, suggesting that CLDN11 has a tumor suppressive role. However, the underlying mechanism remains unclear.

In this study, we observed that the methylation percentage of the CLDN11 promoter inversely correlated with the CLDN11 expression in NPC tumors. Aberrant DNA methylation of the CLDN11 promoter prevents the binding of the transcription activator GATA1 near the transcription start site, resulting in gene silencing. We also dissected CLDN11 protein domains responsible for the inhibition of cell migration function. Two cellular tubulins TUBA1B and TUBB3 were identified to be the novel proteins interacting with CLDN11. The interaction between CLDN11 and these tubulins is necessary for the CLDN11-mediated cell migration inhibition in NPC cells. Tubulins are the basic subunits of microtubules of which form structural network of cytoskeleton in 


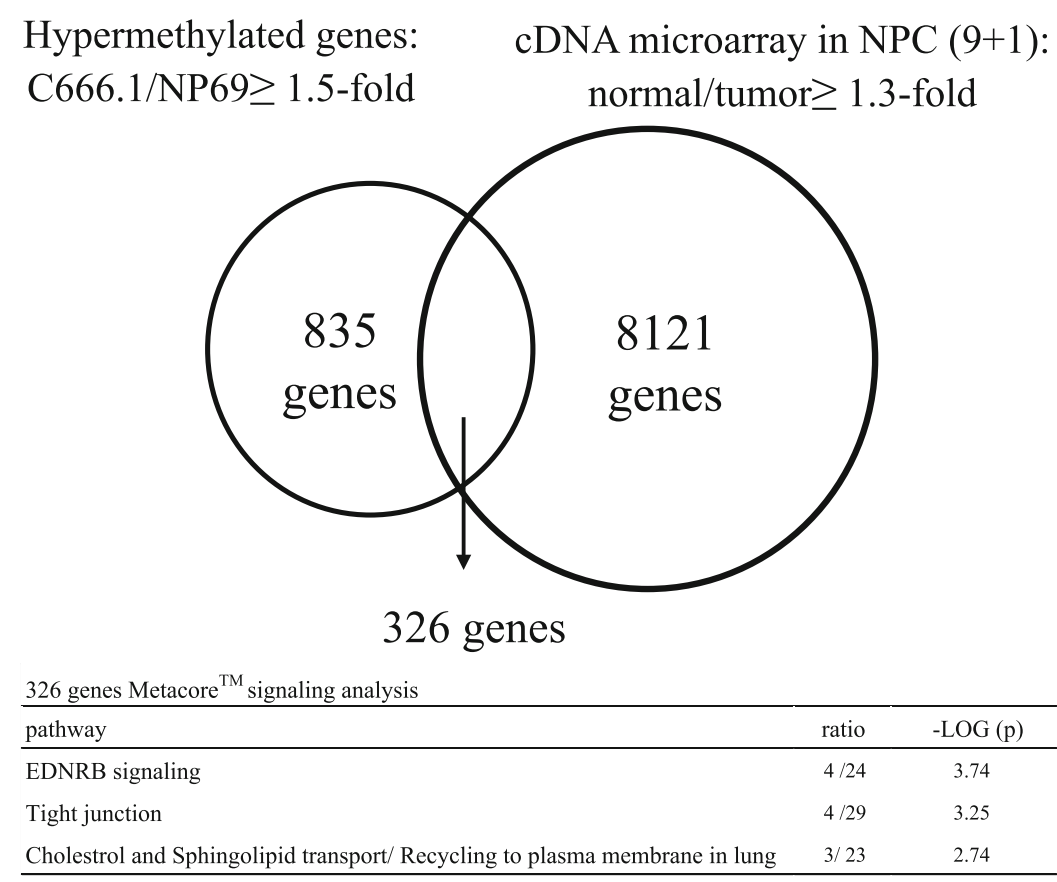

\begin{tabular}{clccccc}
\multicolumn{2}{l}{ Differentially methylated genes involved in tight junction pathway } \\
\hline \multirow{2}{*}{ Gene Symbol } & \multicolumn{1}{c}{ Description } & \multicolumn{2}{c}{ MBD ChIP-seq } & & \multicolumn{2}{c}{ cDNA microarray } \\
\cline { 3 - 4 } & $\begin{array}{c}\text { Methylated DNA } \\
\text { enrichment } \\
\text { (C666.1/NP6) }\end{array}$ & -LOG(q) & & $\begin{array}{c}\text { Fold-Change } \\
\text { (N/T) }\end{array}$ & p-value \\
\hline CLDN11 & claudin 11 & 4.87 & 1693.50 & & 46.34 & $<0.001$ \\
CAV1 & caveolin 1, caveolae protein, 22kDa & 29.75 & 4811.42 & & 2.14 & 0.154 \\
CLDN5 & claudin 5 & 19.58 & 1597.96 & & 1.58 & 0.568 \\
MLLT4 & myeloid/lymphoid or mixed-lineage leukemia & 5.93 & 1572.25 & & 1.39 & 0.074 \\
\hline
\end{tabular}

Fig. 1 Screening for potential hypermethylated genes in NPC. The Venn diagram indicates intersected 326 genes that are both hypermethylated in NPC cells with relative methylated DNA enrichment $\geq 1.5$-fold in C666.1 compared with that of NP69 (1161 genes) and downregulated at least 1.3-fold in nine NPC tumors (T) compared with pooled adjacent normal tissues (N) (8447 genes). The intersected genes were analyzed by MetaCore ${ }^{\mathrm{TM}}$ GeneGo pathway analysis. The top three significant pathways are listed. The bottom table shows the relative methylated DNA enrichment and the expression fold-change of the four genes involved in the tight junction pathway

cytoplasm. It has been known that polymerization and depolymerization of tubulins are essential for cellular processes such as cell movement, transportation of vesicles and organelles, centrosome assembly, and segregation of chromosomes during mitosis and meiosis. Cellular proteins interacting with microtubules are known as microtubule associated proteins (MAPs) [24-26]. The biological significance of the interaction between CLDN11 and tubulins will be further discussed.

\section{Methods}

\section{Cell lines, NPC tissue samples}

The NPC cell lines (C666.1 and HK1) and immortalized normal NP cells (NP69) were gifts from Dr. S. W. Tsao (Hong Kong University, SAR, China). NPC cells were cultured in $10 \%$ fetal bovine serum (FBS) (Thermo Fisher Scientific/Gibco, Waltham, MA)/RPMI1640; NP69 cells were maintained in defined keratinocyte-SFM with growth supplement (Thermo Fisher/ Gibco10744019). The TW02 and TW06 cells were established by Dr. C. T. Lin (National Taiwan University, Taiwan) and cultured in 10\% FBS/DMEM (Thermo Fisher/ Gibco). NPC cells were treated with $10 \mu \mathrm{M}$ demethylation agent 5'Aza for 5 days, with daily replacement of fresh medium. Seven pairs of frozen NPC tumor and adjacent normal biopsies $(<2 \mathrm{~mm})$ were collected from Chang Gung Memorial Hospital by Dr. K. P. Chang. Genomic DNA and RNA of NPC biopsies were simultaneously extracted by using the TRIZOL reagent (Thermo Fisher/Invitrogen) (Additional file 1).

\section{DNA transfection}

TW02 $\left(4 \times 10^{5}\right)$ or HK1 $\left(8 \times 10^{5}\right)$ cells were seeded in 6well plate $\sim 16 \mathrm{~h}$ prior to DNA transfection. Cells were $60 \sim 80 \%$ confluent at the time of transfection. DNA $(2 \mu \mathrm{g})$ and transfection reagent $(4 \mu \mathrm{l})$ jetPRIME (Polyplus, France) were diluted in jetPRIME buffer 
$(200 \mu \mathrm{l})$. The mixture was incubated at room temperature for $10 \mathrm{~min}$ and was added dropwise into the medium. Transfection medium was replaced after $5 \mathrm{~h}$ and cells were harvested $48 \mathrm{~h}$ post transfection.

Construction of luciferase reporters and expression vectors Genomic DNA from NP69 was used as template for the amplification of the promoter regions of CLDN11 $(-900 \sim+197 ;-477 \sim+197 ;-213 \sim+197$ and $-10 \sim+$ 197). PCR products were then subcloned into BglII and HindIII restriction enzyme sites of pGL3Basic (Promega, Madison, WI). Mutant primers were used to generate the GATA sites directed mutants (-92/ HindIII; -62/HindIII; and $+184 / \mathrm{KpnI})$ of CLDN11 promoter luciferase reporters. RNA from normal human peripheral blood cells was reverse transcribed to cDNA. The full length and deletion CLDN11 cDNA clones were PCR-amplified and subcloned into the BamHI and HindIII sites of the pCMV-3tag-8 vector (Stratagene, La Jolla, CA) to generate different Flagtagged clones.

\section{Bisulfite sequencing}

Genomic DNA from NPC patients and cell lines (C666. 1, HK1, NP69) with or without 5'Aza treatment were purified by using Genomic DNA mini Kit (Thermo Fisher/Invitrogen) and 1.8 $\mu \mathrm{g}$ DNA was treated with sodium bisulfite by using a Zymo DNA Modication Kit (Zymo Research, Hornby, Canada) and amplified by specific bisulfite sequencing (BS) primers that were designed by using Methprimer (http://www.urogene.org/ methprimer/, UCSF) [27] and listed in Additional file 2: Table S1. The amplified PCR products were ligated into yT\&A vector (Yeastern Biotech, Taiwan). Eight individual clones were sequenced to determine promoter methylation status.

\section{RNA extraction and quantitative real-time PCR}

Total RNA was extracted and DNaseI treated. Reverse transcription (RT) was performed by using Improm-II reverse transcriptase (Promega) according to the manufacturer's protocol. Q-RT-PCR was performed by using SYBR master mixture (Kapa Biosystems, Woburn, MA) on an IQ5 system (Bio-Rad, Hercules, CA), according to the manufacturer's instructions. The relative gene expression level was determined with respect to that of the $\beta$-actin, and calculated by the $2^{-\Delta \mathrm{Ct}}$ method.

\section{Promoter analysis and luciferase assay}

To normalize each transfection reaction, a renilla reporter plasmid (20 ng pRL-CMV; Promega) was cotransfected with the luciferase reporters $(2 \mu \mathrm{g})$. Cells were harvested $48 \mathrm{~h}$ post-transfection and lysed, and promoter activity was assayed by using the Dual
Luciferase Assay System (Promega) on a GLOMAX 20/ 20 Luminometer (Promega).

\section{DNA pull-down assay}

Double-stranded biotinylated probes (100 pmol) were incubated with $50 \mu \mathrm{l}$ M-280 Streptavidin Dynabeads (Thermo Fisher/Invitrogen) in $5 \mathrm{mM}$ Tris- $\mathrm{HCl} \mathrm{pH} \mathrm{7.5,}$ $0.5 \mathrm{mM}$ EDTA, $1 \mathrm{M} \mathrm{NaCl}$ for $1 \mathrm{~h}$. Nuclear extracts overexpressing flag-tagged GATA1-3F and GATA2-3F $(100 \mu \mathrm{g})$ were precleared by binding to Streptavidin Dynabead $(25 \mu \mathrm{l})$ followed by incubation with the probebounded beads. The Dynabead-bound complexes were washed six times with binding buffer containing $0.5 \%$ NP40. DNA-bound proteins were eluted in SDS sample buffer and verified by Western blotting.

\section{Electrophoretic mobility shift assay (EMSA) \\ EMSA was performed as described previously [9].}

\section{Cell proliferation, migration and invasion assay}

Transient transfected CLDN11 and vector control of TW02 $\left(5 \times 10^{4}\right)$ or HK1 $\left(2 \times 10^{5}\right)$ cells were seeded in 6well plates, and cell numbers were counted daily for 5 days. Cell proliferation was determined by trypan blue (Thermo Fisher/Gibco) exclusion and cell counting. Cell migration and invasion were performed by using the $8 \mu \mathrm{m}$ pore size BD Falcon 24-well insert system and BD Matrigel BioCoat (Corning, NY), respectively. For migration assays, TW02 cells $\left(4 \times 10^{5}\right)$ or HK1 $\left(8 \times 10^{5}\right)$ in 0 . $5 \mathrm{ml}$ serum free medium were seeded to the upper chamber with the lower chamber containing 10\% FBS/ medium $(0.8 \mathrm{ml})$ and cultured for $24 \mathrm{~h}$, allowing cells to migrate through the $8 \mu \mathrm{m}$ membrane. TW02 or HK1 cells were treated with or without tubulin polymerization inhibitor nocodazole (Sigma). For invasion assays, TW02 cells $\left(4 \times 10^{5}\right)$ or HK1 $\left(8 \times 10^{5}\right)$ were seeded to the upper chamber coated with Matrigel with the lower chamber containing $10 \% \mathrm{FBS} /$ medium and cultured for $24 \mathrm{~h}$ (TW02) or $72 \mathrm{~h}$ (HK1). The inserts were stained with $10 \mathrm{mg} / \mathrm{ml}$ crystal violet in $70 \%$ ethanol and $2 \%$ formaldehyde and destained with tap water. The membrane was then excised and stained cells from 10 microscopic fields were counted. All experiments were repeated at least three times.

\section{Antibodies and recombinant proteins}

Primary antibodies: Flag antibodies (M2, Sigma and MDBio, Taiwan), Actin monoclonal antibody (Millipore, Billerica, MA, USA), GATA1 antibody (MDBio), CLDN11 antibodies (Western/ab53041, Abcam, Cambridge, UK; IHC/SC25711, Santa Cruz, Dallas, TX), Tubulin alpha IB (ab108629, Abcam) and Tubulin beta III antibodies (ab52623, Abcam). Secondary antibodies: HRP-conjugated goat anti-rabbit and goat anti-mouse 
antibodies (Gene Teks) were used. Western blot results were visualized by ECL detection kit (Immobilon, Merck/Millipore, Germany). Recombinant proteins GST-GATA1 (H00002623, Abnova, Taiwan) and GSTCLDN11 (H00005010, Abnova) were used in EMSA and tubulin polymerization assay, respectively.

\section{Immunofluorescence and immunohistochemical staining}

TW02 cells $\left(1 \times 10^{5}\right)$ were grown on polylysine-coated coverslip for $24 \mathrm{~h}$ and followed by transfection of pCMV/CLDN11-3F or vector control plasmids. Posttransfection $24 \mathrm{~h}$, cells were fixed with $4 \%$ formaldehyde, permeabilized with $0.1 \%$ Triton X-100/PBS and blocked with $5 \%$ BSA/PBS. The coverslips were incubated with the primary antibodies for $2 \mathrm{~h}$ at room temperature, followed by incubation with appropriate FITC- or rhodamine-conjugated secondary antibodies (Jackson ImmunoReasearch, West Grove, PA). Finally, the nuclei were stained with DAPI fluorescent dye (AAT Bioquest, Sunnyvale, CA). Coverslips were mounted with the VECTASHEILD reagent (Vector Laboratories, CA). The images were acquired by confocal microscopy (Zeiss, LSM780), CGU, Microscope Center. Immunohistochemistry was performed by CGU, Pathology Core Laboratory by using NPC tissue array (NPC961; Pantomics, Richmond, CA).

\section{Statistical analysis}

Values on bar graphs and curves were displayed as mean \pm standard deviation (SD). For MBD ChIP-seq analysis, data were expressed as fold-enrichment of normalized experiment tags over normalized background tags within regions and compared by false discovery rate adjusted $p$ value (q value) and analyzed by the DNAnexus platform (DNAnexus, CA, USA). For NPC microarray analysis, gene expression in 9 NPC tumor was compared with that of "one-combined non-tumors" by using the one-way analysis of variance (ANOVA) by Partek Genomics Suite (Partek, MO, USA). Intergroup comparisons were conducted by using the unpaired two-sample $t$ test (Fig. 2, Fig. 3, Fig. 4, Fig. 5) or paired two-sample $t$ test (Fig. 2b). A $p$-value less than $0.05(*), 0.01(* *), 0.001(* * *)$ was considered statistically significant. All graphed plots and statistical analyses (Fig. 2, Fig. 3, Fig. 4, Fig. 5) were conducted by the Prism 5 software (GraphPad) .

\section{Results}

Tight junction CLND11 is a potential hypermethylated and downregulated gene in NPC

Two gene lists (1) differentially methylated genes (1161) and (2) downregulated genes (8447) were intersected. There were 326 gene candidates identified as potential genes inactivated because of aberrant DNA methylation (Fig. 1). To further understand the contribution of the inactivation of these genes toward NPC carcinogenesis, we further analyzed them by using gene ontology pathway analysis software MetaCore (Thomson Reuters, Toronto, Canada). The tight junction pathway is a significant pathway involved in cell adhesion $(-\log P=3.25$; Fig. 1). The tight junction-related gene $C L D N 11$ was selected because the relative CLDN11 methylated DNA enrichment was high (4.87-fold) in NPC cells (Fig. 1 and Additional file 3: Figure S1) and the CLDN11 mRNA expression reduction fold-change in NPC tissues was also high (46.34-fold, Normal/Tumor; Fig. 1), suggesting that CLDN11 is hypermethylated and downregulated in NPC.

\section{CLDN11 methylation status is conversely correlated with its expression in NPC}

To investigate the differentially methylated CpG sites of the CLDN11 promoter in clinical samples, we performed bisulfite sequencing analysis of seven paired tumor and nontumor genomic DNA ranging from -137 to +405 containing $52 \mathrm{CpG}$ sites. The results showed that CLDN11 promoter was hypermethylated in seven NPC tumor tissues (34.62\%-85.34\%, average 62.64\%) compared with that of the paired adjacent normal tissues $(1.20 \%-42.07 \%$, average 10\%; Fig. 2a). The methylation percentage of the same promoter region was also higher in the NPC cells C666.1 (99.28\%) than that of immortalized normal NP cells NP69 (1.44\%), indicating that CLDN11 is hypermethylated in NPC tumors and cells.

To correlate the methylation status with the mRNA expression level, we performed qRT-PCR to examine CLDN11 mRNA expression levels in the same NPC tissues and in various NPC cells. The data demonstrated that CLDN11 mRNA expression was significantly reduced (4.3-53.5-fold, average 22.5-fold) in NPC tissues versus adjacent normal tissues (Fig. $2 b, P=0.004$ ). Similarly, CLDN11 mRNA expression levels decreased 15-50-fold in NPC cells (C666.1, HK1, TW02 and TW06) compared with those in NP69 cells (Fig. 2c). Furthermore, CLDN11 mRNA expression levels were restored upon DNA methylation inhibitor, 5'Aza, treatment in the three NPC cells C666.1, HK1, and TW02, but not in TW06 (Fig. 2d), suggesting that transcriptional repression of CLDN11 by DNA methylation is occurred in NPC cells.

We next performed immunohistochemical staining by using NPC tissue array (Pantomics, Richmond, CA) to examine the CLDN11 protein expression levels in NPC. Seven of nine NPC tumors had lower CLDN11 expression levels (Fig. 2e, $\mathrm{T}<\mathrm{N} \# 1-7$ and Additional file 4: Figure S2) compared with their normal counterparts; by contrast, two NPC tumors (Fig. 2e, $\mathrm{T}=\mathrm{N} \# 8-9$ and Additional file 4: Figure S2) had similar CLDN11 expression compared with the adjacent normal tissues. In addition to the reduction in CLDN11 expression, NPC tumor cells were 
a
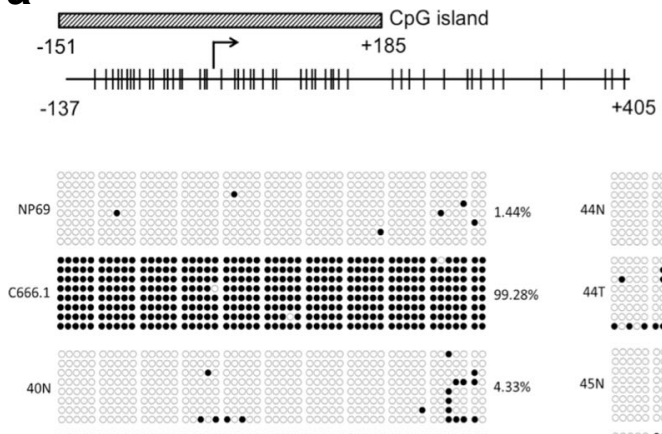

${ }_{40}$ :

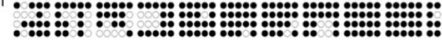

$41 \mathrm{~N}$

:.: $3.85 \%$

$\because$.

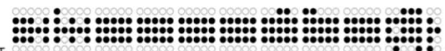

${ }^{41}$

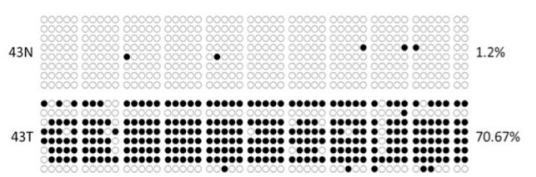

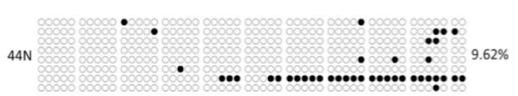

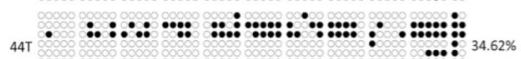
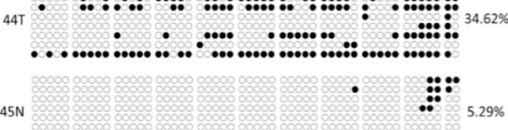

(1)

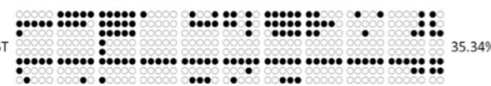

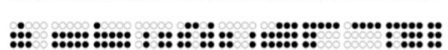

${ }^{48 N}$

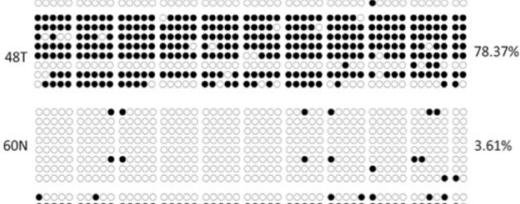

600

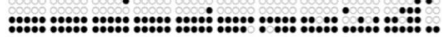

b
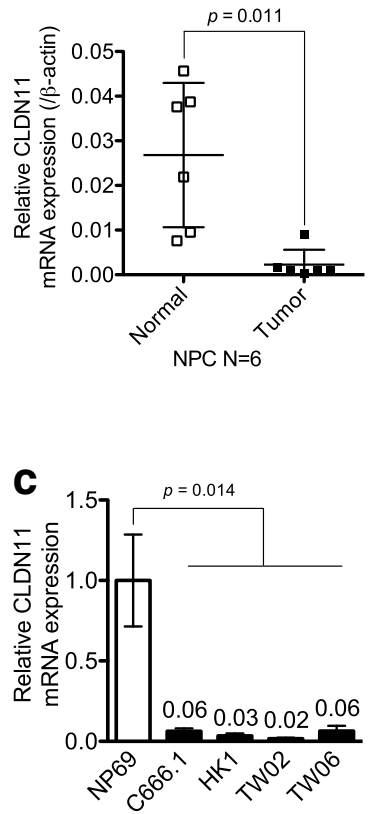

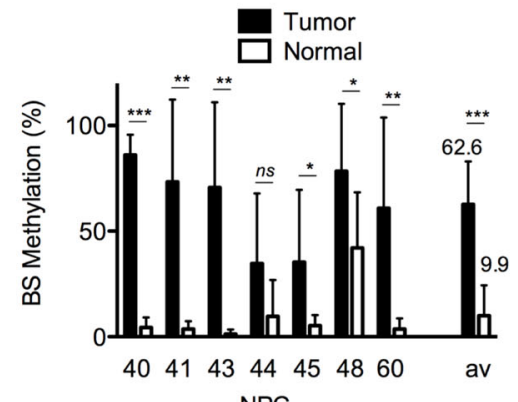

NPC

e

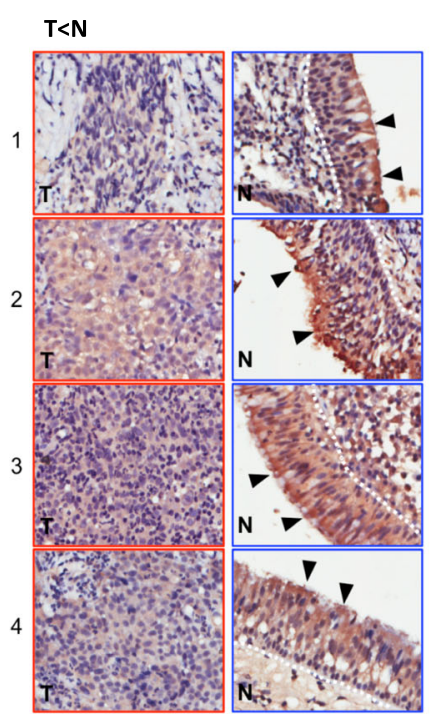

d

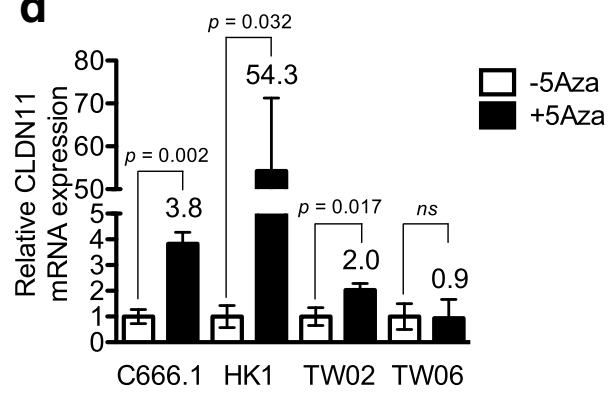

C666.1 HK1 TW02 TW06

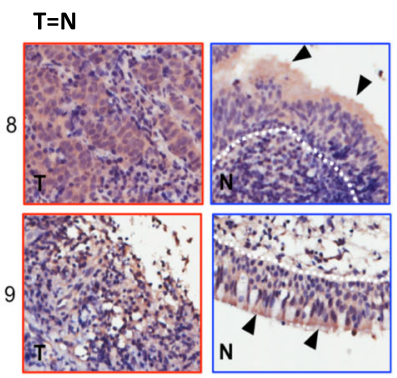

Fig. 2 (See legend on next page.)

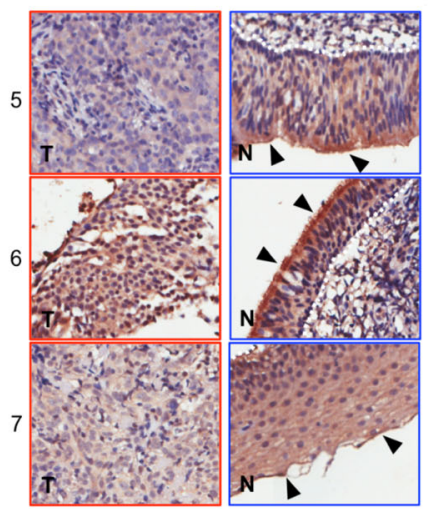


(See figure on previous page.)

Fig. 2 Identification of the hypermethylated and downregulated gene CLDN11 in NPC. a Bisulfite sequencing analysis was performed on - 137 to +405 in C666.1 and NP69 cells, and seven paired NPC clinical samples. Each horizontal row represents a single clone; the methylation percentages of at least eight individual clones are indicated as unmethylated (o) and methylated (•) CpG sites. The lower panel shows the average methylation percentage for each sample. qRT-PCR analysis of CLDN11 mRNA expression was performed in (b) 6 paired NPC tissues and (c) NP69 cells and four NPC cell lines. The results were normalized to $\beta$-Actin expression. $\mathbf{d}$ Columns represent the relative fold-change of the restored CLDN11 mRNA expression normalized with respect to $\beta$-Actin expression in NPC cell lines with (+) or without (-) $10 \mu \mathrm{M}$ 5'Aza treatment. e Immunohistochemistry staining analysis of CLDN11 protein expression in nine paired NPC tissue arrays (Pantomics). Tumor tissues (T) and the corresponding adjacent normal tissues (N) are indicated. The results are shown at 200x magnification (CLDN1 1 staining intensity $T<\mathrm{N}: 7$ pairs, $T=\mathrm{N}: 2$ pairs). Higher magnification is shown in Additional file 4: Figure S2. White dotted lines mark the border of basement membrane of normal epithelial cells; black triangles indicate the apical membranous staining signals of CLDN11

morphologically distinct from the normal epithelial cells, becoming disorganized and having irregular cell boundary with large nuclei. A paired normal tissue $\left(\begin{array}{ll}\# 7 & \mathrm{~N}\end{array}\right)$ already demonstrated hyperplasia condition (presence of $\geq 4$ cell layers above the basement membrane); nevertheless, CLDN11 expression remained relatively higher compared with its tumor tissue. Thus, our data supported that CLDN11 methylation status is conversely correlated with mRNA expression and that DNA hypermethylation is responsible for the reduction in CLDN11 mRNA and protein expression levels in NPC.

\section{DNA methylation causes CLDN11 inactivation by interfering with GATA binding toward the CLDN11 promoter}

To study the underlying molecular mechanism by which methylation inactivates CLDN11 transcription, a series of deletion CLDN11 promoter luciferase reporters were constructed and the transcriptional regulation of CLDN11 and the key transcription factors involved were clarified (Additional file 5: Figure S3). Luciferase assay results indicated that in TW02 and HK1 cells, the minimal CLDN11 promoter sequences are located within -213 to $+197, p C L D N 11(-213)$ (Fig. 3a). We then predicted transcription factor binding sites within the CLDN11 minimal promoter on the basis of the TFSEARCH website and selected transcription factor binding consensus, which overlapped with previous differentially methylated CpG sites. Consequently, GATA1 and GATA2 were the prominent transcription regulators for $p C L D N 11$. To investigate whether GATA1 or GATA2 transactivates CLDN11 promoter activity, each GATA expression plasmid and $p C L D N 11(-213)$ were cotransfected into TW02 and HK1 cells, separately. Elevated $p C L D N 11(-213)$ promoter activities were detected after ectopically expressing GATA1 or GATA2 (Fig. 3b), suggesting that both GATA1 and GATA2 act as transcriptional activators for the CLDN11 promoter. Three putative GATA-binding motifs are present within the minimal CLDN11 promoter: GATA1 site ( 92 to 83), GATA1 and 2 site ( -62 to -53$)$, and GATA2 site $(+184$ to +193$)$ (Fig. 3c). To characterize which site is responsible for CLDN11 promoter activation, we mutated each GATA consensus sequences as shown in Fig. 3c (upper panel; S1, GATA1 site: CGCGATTGGC to CAAGCTTGGC; S2, GATA1/2 site: GCCGATTGGT to GAAGCTTGGT; S3, GATA2 site: GGACATCCTG to GGACGGTACC). The activity of the GATA-mutated promoters decreased to $\sim 50 \%$ in $\mathrm{S} 1$ and $\mathrm{S} 3$ and significantly reduced to $5 \%$ in S2 compared with that of the wild type $p C L D N 11(-213)$ in both TW02 and HK1 cells (Fig. 3c), indicating that GATA1 and GATA2 activate the CLDN11 promoter mainly through $\mathrm{S} 2$, a GATA1/2 site situated at a close proximity to the transcription start site.

To determine whether the binding of GATA1 and GATA2 toward the GATA1/2 site is interfered by DNA methylation, we performed DNA pull-down assays by using wild type (WT), methylated (ME), and mutated (MT) biotinylated probes containing GATA1/2 site $(-67$ to -47 ) and nuclear extracts from TW02 cells overexpressing either FLAG-tagged GATA1-3F or GATA2-3F. Both DNA methylation and mutation of GATA consensus sequences reduced the binding of either GATA1-3F or GATA2-3F when compared with that of the WT probes (Fig. 3d). However, the degree of inhibition of GATA1-3F binding is slightly greater than that of GATA2-3F toward the same methylated probe. Similarly, we used the same probes to bind purified recombinant GST-GATA1 and performed electrophoretic mobility shift assay (EMSA) experiments. Consequently, the recombinant GATA1 bound well to the WT probe but poorly to the methylated probe; less than $\sim 10 \%$ of the binding was detected in the methylated probe (Fig. 3e). These data indicated that methylation impairs the binding of transcription activator GATA1 and leads to transcriptional inactivation of CLDN11 in NPC.

\section{CLDN11 inhibits cell migration and invasion in NPC cells}

Silencing of TSGs through promoter hypermethylation is an early event during cancer formation [2]. To characterize the biological roles of CLDN11 in NPC, we performed cell proliferation, migration, and invasion assays in TW02 and HK1 cells transiently overexpressed with FLAG-tagged CLDN11-3F or the vector control. CLDN11 did not affect the cell proliferation 


\section{TW02}

a

b

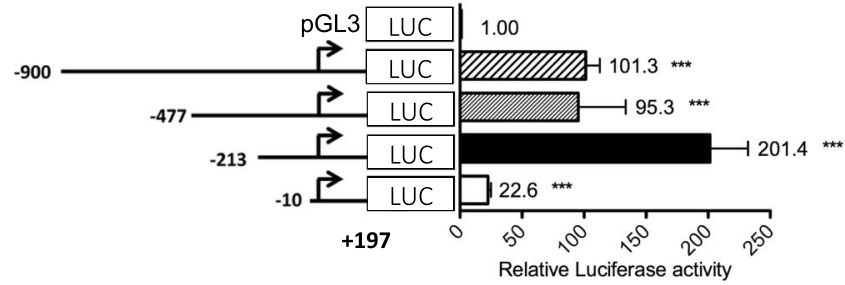

$-213$

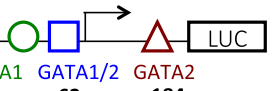

GATA1 GATA1/2 GATA2
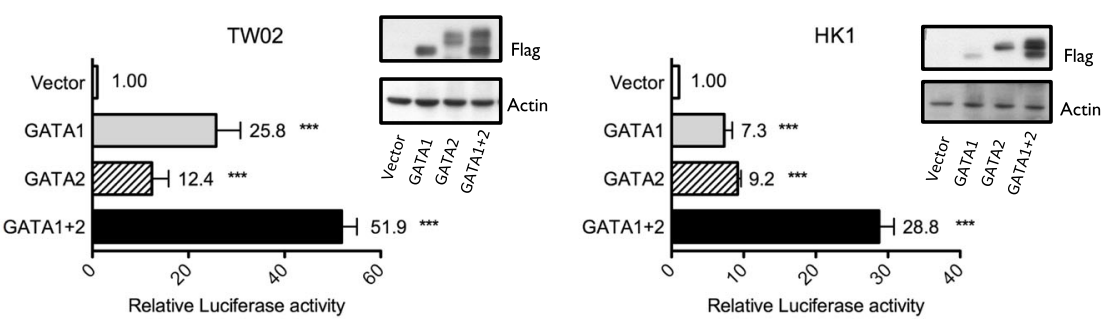

C

S1

S2

S3

-92 GATA1 $\bigcirc_{-83}$

GATA1/2

$\square_{-53}$

$+184$

GATA2 $\triangle$

pGL3| 1.00

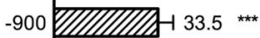

-477 .

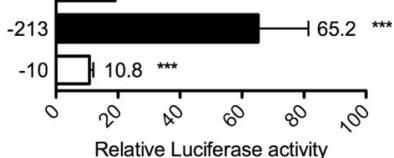

WT--CCCGCGATTGGC C---CGCCGATTGGT---GGGGACATCCTGGCG-

MU--CCCAAGCTTGGC $C$--_GAAGCTTGGT $\mathrm{G}---\mathrm{GGGGACGGTACC} \mathrm{GCG}$ -

TW02

HK1
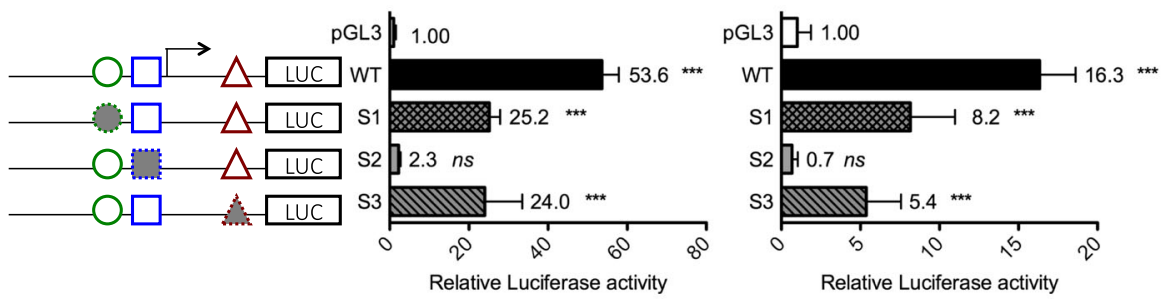

d

GATA1/2

$$
----\rightarrow
$$

WT: CTGCCGCCGATTGGTGCTCGC

ME : CTGCCGCCGATTGGTGCTCGC

MT: CTGCCGAAGCTTGGTGCTCGC

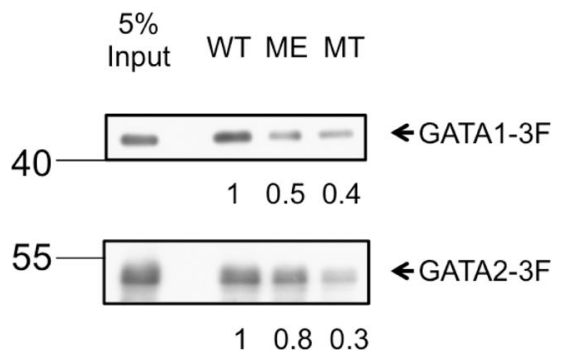

e

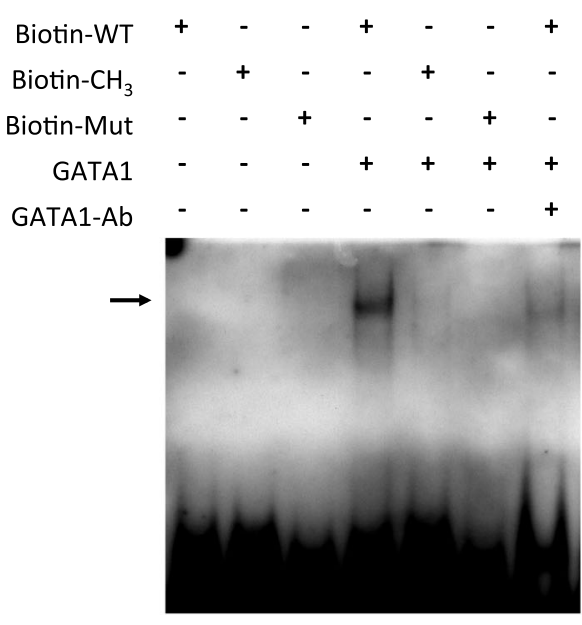

10.1

Fig. 3 (See legend on next page.) 


\begin{abstract}
(See figure on previous page.)
Fig. 3 Methylation inhibits CLDN11 promoter activity by impairing the binding of the transcriptional activator GATA1. a Different promoter activities of a series of CLDN11 promoter deletion luciferase reporters were assayed in TW02 and HK1 cells. Intergroup comparison was conducted relative to the empty vector (pGL3). b The minimal CLDN11 promoter reporter $(-213$ to +197$)$ and the FLAG-tagged GATA1 and GATA2 expression clones were cotransfected into TW02 and HK1 cells. Three putative GATA binding sites: GATA1 (- 92), GATA1/2 (- 62), and GATA2 (+ 184), are indicated. GATA1: GATA1 overexpression, GATA2: GATA2 overexpression, GATA1 + 2: GATA1 and GATA2 co-overexpression. Intergroup comparison was conducted relative to the vector control. The expression levels of ectopic GATA-1, GATA-2, and actin (internal control) were examined through Western blotting. c Minimal CLDN11 promoter reporters with wild-type (WT) and three mutated GATA binding sites (MU, S1-S3) were transfected into TW02 and HK1 cells, respectively. Intergroup comparison was conducted relative to the empty vector (pGL3). a $\sim$ c All the luciferase reporter activities were normalized with respect to renilla activity. $\mathbf{d}$ DNA pull-down assay was used to analyze the binding affinity of exogenous FLAG-tagged GATAs (GATA1-3F and GATA2-3F) to WT, methylated (ME), and mutated (MT) biotinylated probes containing GATA1/2 site; the sequences of these probes are shown in the upper panel. Methylated cytosine is indicated by using " $m$ " above $C$, and mutated sequences are underlined. Anti-GATA1 and anti-FLAG antibodies were used for examining the amount of bound GATA1-3F and GATA2-3F in the immunoprecipitates and 5\% input. e EMSA was performed to compare the binding affinity of purified recombinant GATA1 for WT, ME, and MT biotinylated probes of the GATA1/2 site. The arrow indicates DNA-protein complexes. Antibodies against GATA1 were used for supershift assays
\end{abstract}

ability (Fig. 4a) but significantly reduced cell migration $(\sim 40 \%)$ and invasion $(\sim 50 \%)$ abilities compared with the vector control in NPC cells (Fig. 4b), demonstrating that CLDN11 inhibits cell migration and invasion in NPC cells.

\section{Intracellular loop and C-terminus of CLDN11 are required for TUBA1B and TUBB3 interaction and CLDN11-mediated anti-migration}

To understand the mechanism of CLDN11-mediated migration suppression, we identified the interacting proteins of CLDN11. We performed co-immunoprecipitation assays of FLAG-tagged CLDN11 (C) and vector control (V) by using FLAG M2 beads. The precipitated proteins were resolved on SDS-PAGE and stained by using Coomassie blue (Additional file 6: Figure S4). Each lane from the gel was sliced, trypsin-digested, and analyzed through liquid chromatography-tandem MS (LC-MS/MS) (CGU Proteomics Center). The resulting MS and MS/MS spectra were compared with the Human v3.26 database by using the Mascot algorithm. The spectral counting label-free quantification method [28] was used to quantitate the protein samples. Putative CLDN11 interacting proteins (Additional file 7: Table S2) were selected on the basis of the ratio of total spectra numbers of fold-change $(>30)$ enriched in CLDN11 versus control $(P<0.01)$. Significant interacting proteins tubulin alpha-1B (TUBA1B) and tubulin beta-3 (TUBB3) as well as the bait CLDN11 itself were identified; their corresponding spectra numbers of fold-change (C/V) are listed in Fig. 5a (peptide sequences detected are listed in Additional file 8). We subsequently performed co-immunoprecipitation and Western blotting assays to validate whether CLDN11 can interact with endogenous TUBA1B and TUBB3. FLAG-tagged CLDN11$3 \mathrm{~F}$ and vector control were transiently overexpressed in NPC cells individually and used to immunoprecipitate endogenous TUBA1B and TUBB3, respectively. Our data indicated that exogenous CLDN11-3F indeed associated with small amount of cytoskeleton proteins TUBA1B and TUBB3 (Fig. 5b).

We next overexpressed FLAG-tagged CLDN11-3F in TW02 cells and observed the subcellular localization and distribution of the exogenous CLDN11-3F and endogenous cytoskeleton proteins TUBA1B and TUBB3 through confocal microscopy. The fluorescent signal of these proteins was quantitated and analyzed (Fig. 5c). Although the exogenous CLDN11-3F was overexpressed, the measured signal of CLDN11-3F was approximately 10 times lower than that of the endogenous tubulin, reflecting that the difference in the expression levels of CLDN11-3F and tubulins was rather high. Nearly half of the exogenous CLDN11-3F (41\%-48\%) was colocalized with a small fraction of endogenous tubulins, TUBA1B and TUBB3 (4\%-8\%), in the cytoplasm of NPC cells.

To elucidate the interaction domains of CLDN11 for tubulins, various deletion constructs of CLDN11-3F were generated (Fig. 5d). These deletion clones were overexpressed and immunoprecipitated by using Flag M2 beads. We observed that either the intracellular loop or the cytoplasmic C-terminus could contribute to pull down the two endogenous tubulins, suggesting that they are the two crucial domains of CLDN11 interacting with TUBA1B and TUBB3 (Fig. 5d). Subsequently, these deletion clones were introduced into NPC cells to confirm whether the intracellular loop and cytoplasmic Cterminus of CLDN11 are required for CLDN11mediated migration suppression. Notably, in the absence of the tubulin interacting domains (deletion clone no. 4 and 5), CLDN11 lost its ability to block cell migration (Fig. 5e), indicating that these domains are essential for CLDN11 to execute its anti-migration function.

$\alpha$ - and $\beta$-tubulins undergo dynamic polymerization forming microtubules, of which are required for mitosis, cell movement, intracellular transport, and structural support. We then investigated whether the presence of 
a
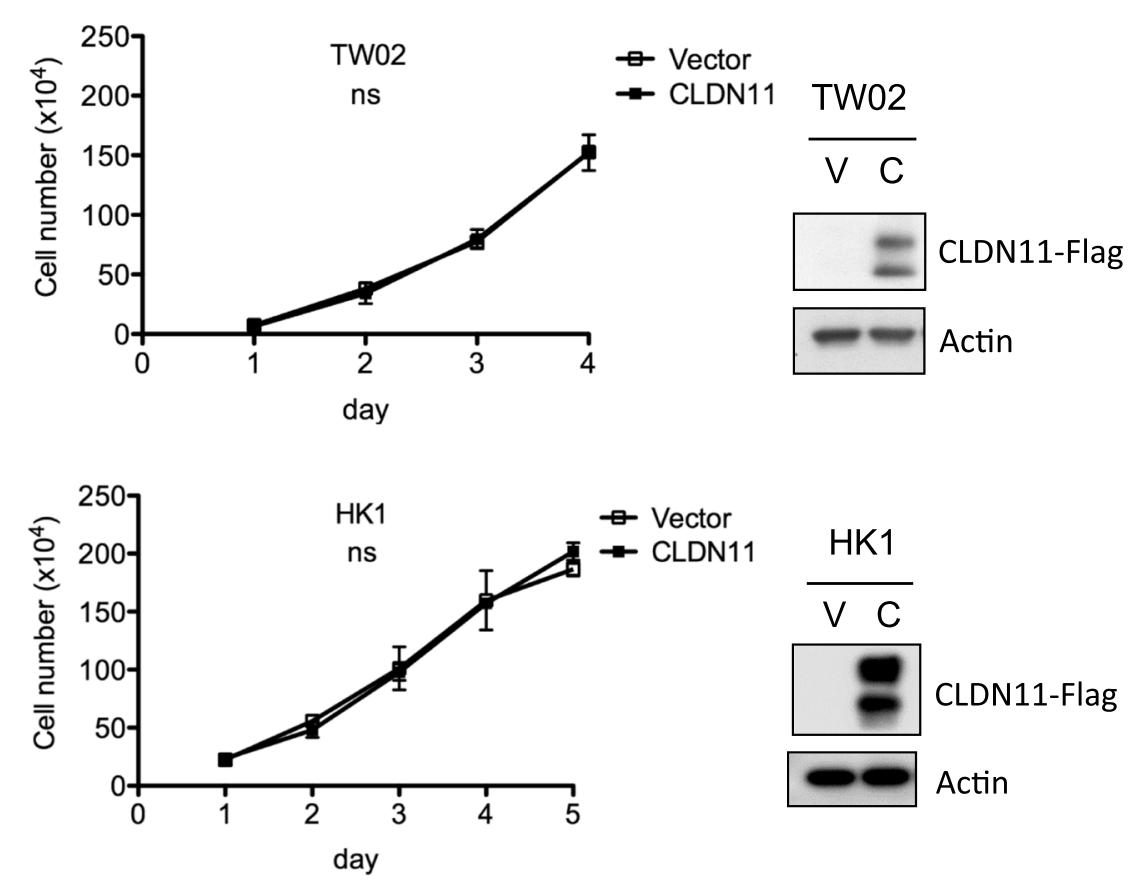

b

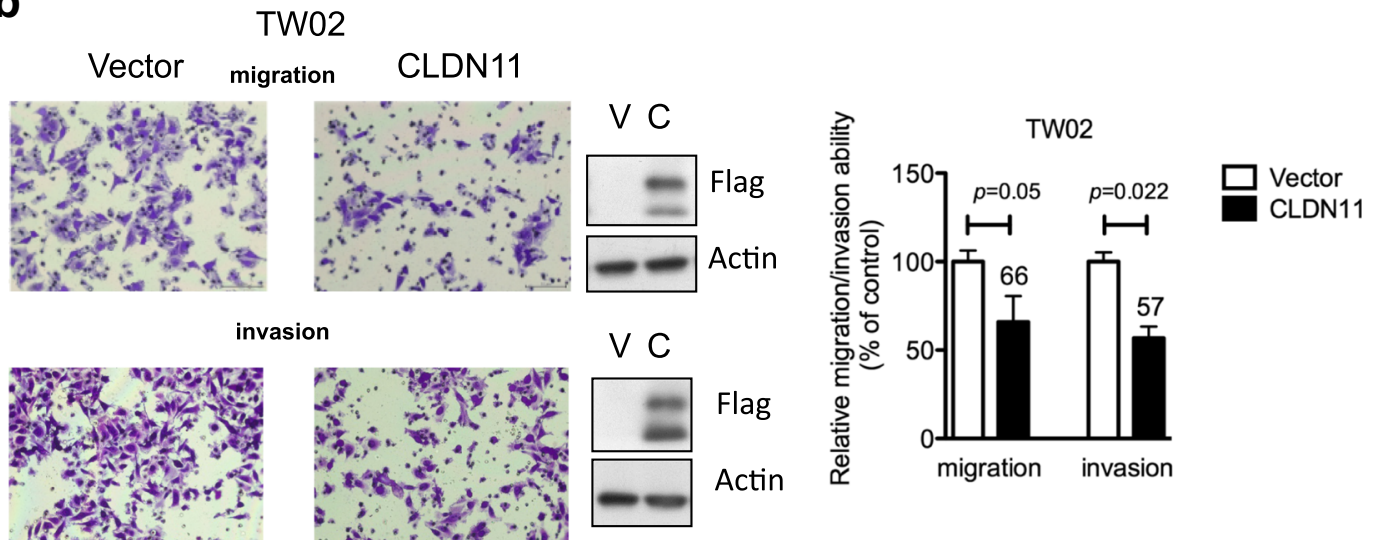

HK1
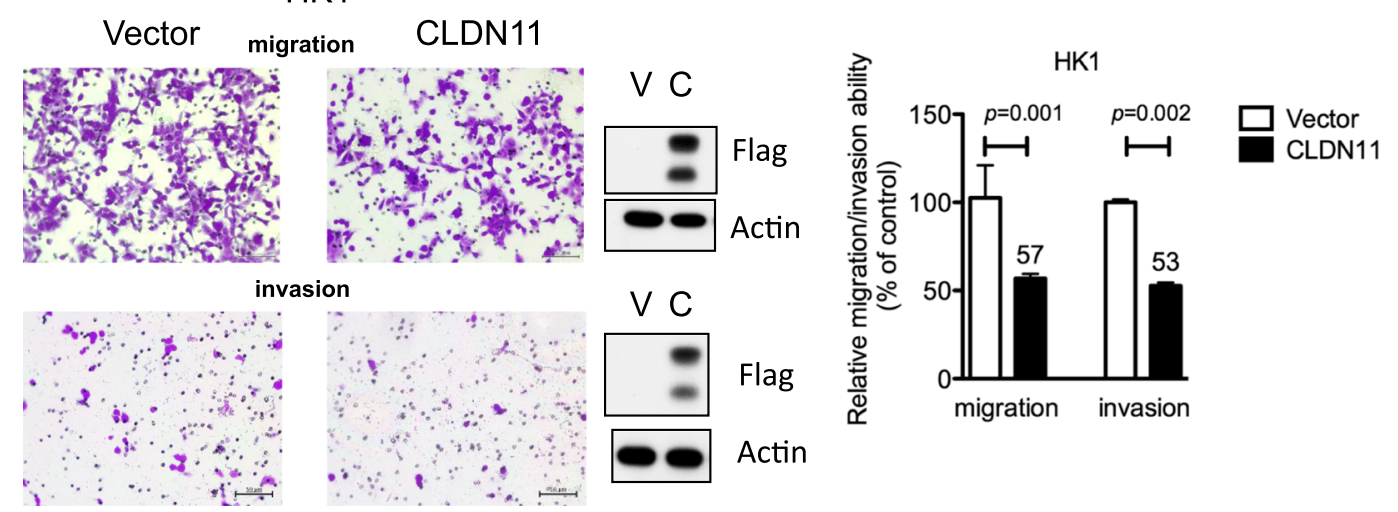

Fig. 4 (See legend on next page.) 
(See figure on previous page.)

Fig. 4 CLDN11 reduces migration and invasion abilities of NPC cells. a Cell number (proliferation) was monitored in TW02 and HK1 cells expressing CLDN11 (C) or vector control $(V)$ for 5 days. b Transwell cell migration and invasion were assayed in TW02 and HK1 cells expressing CLDN11 (C) or vector control (V). Migrated or invaded cells were counted in 10 microscopic fields. The data are presented as percentage of cell migration or invasion relative to control. The expression of FLAG-tagged CLDN11 and actin (internal control) was detected by using specific antibodies (anti-Flag and anti-actin)

CLDN11 affects in vitro tubulins polymerization. By determining the optical density at $340 \mathrm{~nm}$ (OD340) of the reaction, we observed that tubulins rapidly polymerized in the standard reaction. The reaction reached a plateau after 20 min (Additional file 9: Figure S5a) and the Vmax of the reaction was $44.2 \mathrm{mOD} / \mathrm{min}$ at $11.5 \mathrm{~min}$ (Additional file 9: Figure S5b). The addition of a tubulin polymerization inhibitor, nocodazole $(10 \mu \mathrm{M})$, substantially blocked the process; the polymerization reached plateau after $60 \mathrm{~min}$ (Additional file 9: Figure S5a) and the Vmax was $5.6 \mathrm{mOD} / \mathrm{min}$ at $40 \mathrm{~min}$ (Additional file 9: Figure S5b). The presence of GST-CLDN11 affected the tubulin polymerization process. The time required to reach plateau was 40 min for GST-CLDN11 (Additional file 9: Figure S5a), and the Vmax was $16.8 \mathrm{mOD} / \mathrm{min}$ at $24 \mathrm{~min}$ for GST-CLDN11 (Additional file 9: Figure S5b). These results strongly suggested that CLDN11 interferes with tubulin polymerization, that the intracellular loop and Cterminus of CLDN11 are necessary for the binding of TUBA1B and TUBB3, and that the deletion of intracellular loop and C-terminus of exogenous CLDN11 impairs the CLDN11-mediated migration blockage in NPC cells. By interacting with microtubules, CLDN11 essentially maintains and stabilizes normal epithelial cell structure. Thus, the silencing of CLDN11 promotes the migration ability of NPC cells.

To determine whether a tubulin polymerization inhibitor can be used to block the migration ability of NPC cells, we performed migration assays in the presence of nocodazole. This treatment effectively blocked the migration ability of TW02 and HK1 cells in a dose-dependent manner (Fig. 5f) without affecting cell viability (Fig. 5g), thus indicating that the nocodazole treatment results were similar to those of CLDN11 re-expression in NPC cells. Hence, tubulin polymerization inhibitor(s) in general may compensate CLDN11 downregulation, serving as potential therapeutic drug(s) for NPC.

\section{Discussion}

Genome-wide profiling for hypermethylated DNA sequences in tumor relative to nontumor samples facilitates identification of epigenetically misregulated cancerrelated genes [29, 30]. Moreover, hypermethylated genes may serve as diagnostic or prognostic biomarkers for early detection as well as potential therapeutic targets for cancer treatment $[31,32]$. Here, we found that the tight junction CLDN11 is a novel differentially hypermethylated and downregulated gene in NPC tumors. Our bisulfite sequencing analysis demonstrated that the hypermethylated CLDN11 promoter region is located at -137 to +405 , similar to previously reported differentially hypermethylated region in gastric cancer $(-104$ to $+4)$ [23] and in melanoma (+144 to + 249) [33]. This indicates that aberrant hypermethylation of CLDN11 promoter frequently occurs near the transcription start site in different cancers. Based on the promoter assay results, GATA1 and GATA2 are the two crucial transcription activators responsible for CLDN11 activation. A study demonstrated that GATA family proteins upregulate murine CLDN11 promoter activity [34], indicating the GATA-mediated CLDN11 activation is conserved among species. Although no apparent CpG site has been noted within the consensus sequences of GATA1 (G/ CNNGATTNNNN) and GATA2 (NNNGATA/GNNN) on the CLDN11 promoter, two neighboring CpG sites upstream of the GATA1/2 (-62) core consensus have been revealed. The methylation of these flanking $\mathrm{CpG}$ sites may still impede the binding of GATA, causing transcriptional silencing of CLDN11.

CLDN11 is mainly expressed in the brain and testes, where it form tight junction strands in oligodendrocytes and Sertoli cells, respectively [35, 36]. CLDN11-null mice exhibit defects in the neurological and reproductive systems, suggesting the pivotal role of CLDN11 in establishing the paracellular physical barrier of tight junctions necessary for normal CNS function and spermatogenesis [37-39]. In addition to cell barrier functions, CLDN11 appears to have a tumor suppressor function. First, CLDN11 is often inactivated in tumors; nevertheless, its re-expression decreases cell motility and invasiveness in gastric and bladder cancers $[18,23]$, similar to the observations in this study. Normal nasopharynx epithelial cells are uniformly arranged, with 3-4 cell layers attached to the basement membrane. By contrast, NPC cells lose orderly morphology, cell polarity, and the basement membrane, but acquire aberrant cell expansion ability. Our immunohistochemistry data demonstrated strong staining signal for CLDN11 at the apical surface of the normal nasopharynx cells and exhibited typical tight junction staining pattern. However, CLDN11 expression was low and diffusely located in the cytoplasm of NPC tumor tissues. Notably, the forced expression of 


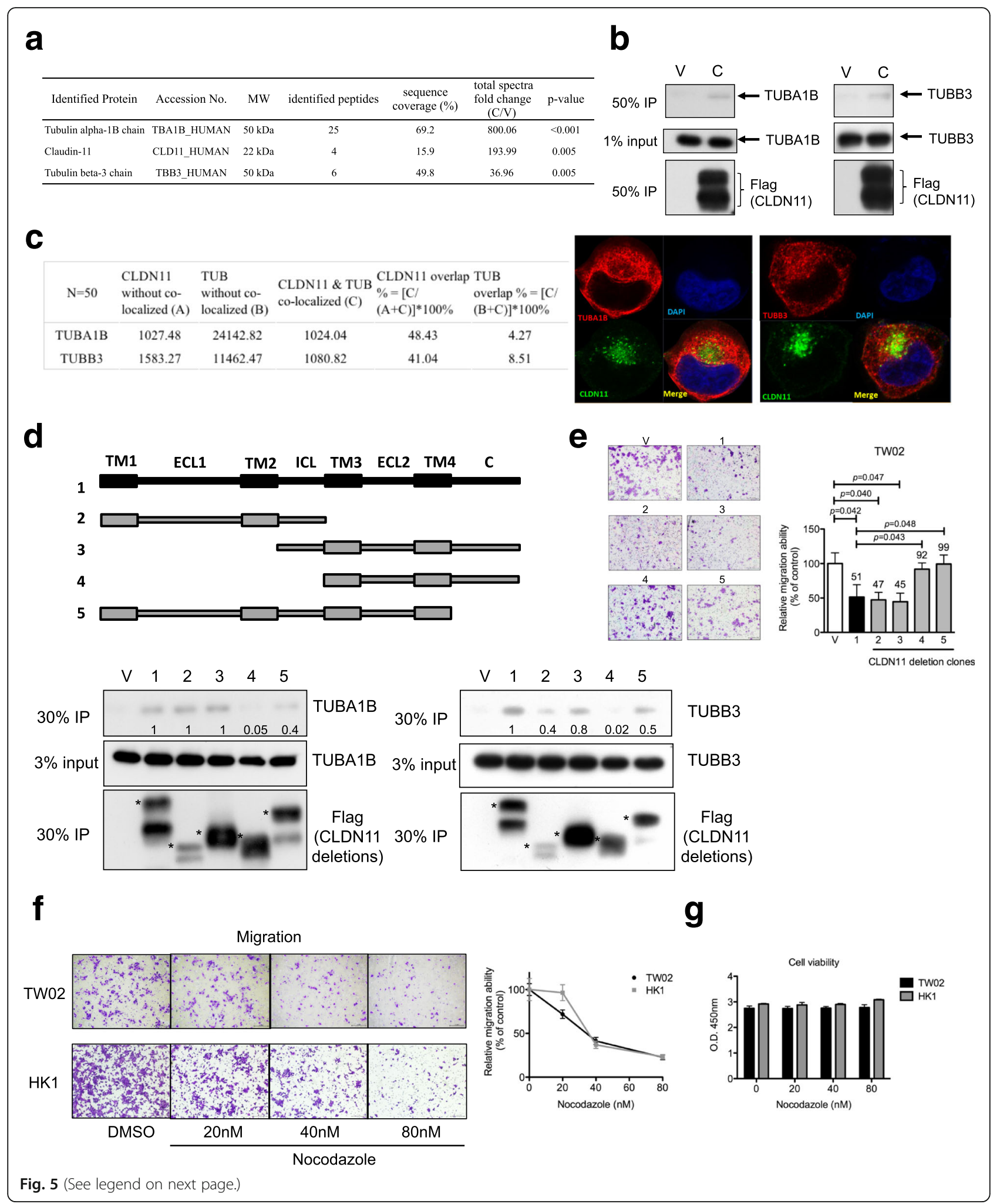


(See figure on previous page.)

Fig. 5 TUBA1B and TUBB3 are the interacting proteins of CLDN11; CLDN11 blocks cell migration by interfering with tubulin polymerization. a TW02 cells transfected with FLAG-tagged CLDN11 construct (C) or vector control plasmid $(V)$ were harvested after 48 h. TW02 cell extracts and M2 beads were used for a co-immunoprecipitation assay. CLDN11-interacting proteins identified through LC-MS/MS and top-ranking proteins are listed in the table. $\mathbf{b}$ Immunoblot analyses were performed to confirm the interaction between FLAG-tagged CLDN11 and endogenous TUBA1B (left panel) or TUBB3 (right panel). c Subcellular distribution of exogenous FLAG-tagged CLDN11 and endogenous TUBA1B or TUBB3 in TW02 cells was assayed through immunofluorescence staining $24 \mathrm{~h}$ after transfection. The fluorescent signal (pixel) of each individual cell was quantitated by using the Zen 2.0 software $(n=50)$. The proportion of colocalized fluorescent signals (\%) is indicated (FLAG-tagged CLDN11, green; Tubulins, red; DAPI, blue). $\mathbf{d}$ Schematic illustrations of WT and four deletion FLAG-tagged CLDN11 molecules-transmembrane (TM), extracellular loop (ECL), intracellular loop (ICL), and C-terminus (C). Various FLAG-tagged CLDN11 deletion clones or a vector control were used to dissect the interacting domains on CLDN11 that are crucial for the interaction of endogenous TUBA1B and TUBB3. Input (3\%) and immunoprecipitates (30\% IP) were assayed through immunoblot analysis by using appropriate antibodies (anti-Flag, anti-TUBA1B and anti-TUBB3). The asterisks denote the major bands of ectopic CLDN11 in the immunoblot assays. e CLDN11 deletion clones were used to perform cell migration assay and to determine which domains on CLDN11 are necessary for blocking cell migration in TW02 cells. $\mathbf{f}$ Cell migration and (g) cell viability assays were performed in the presence or absence of nocodazole in TW02 or HK1 cells

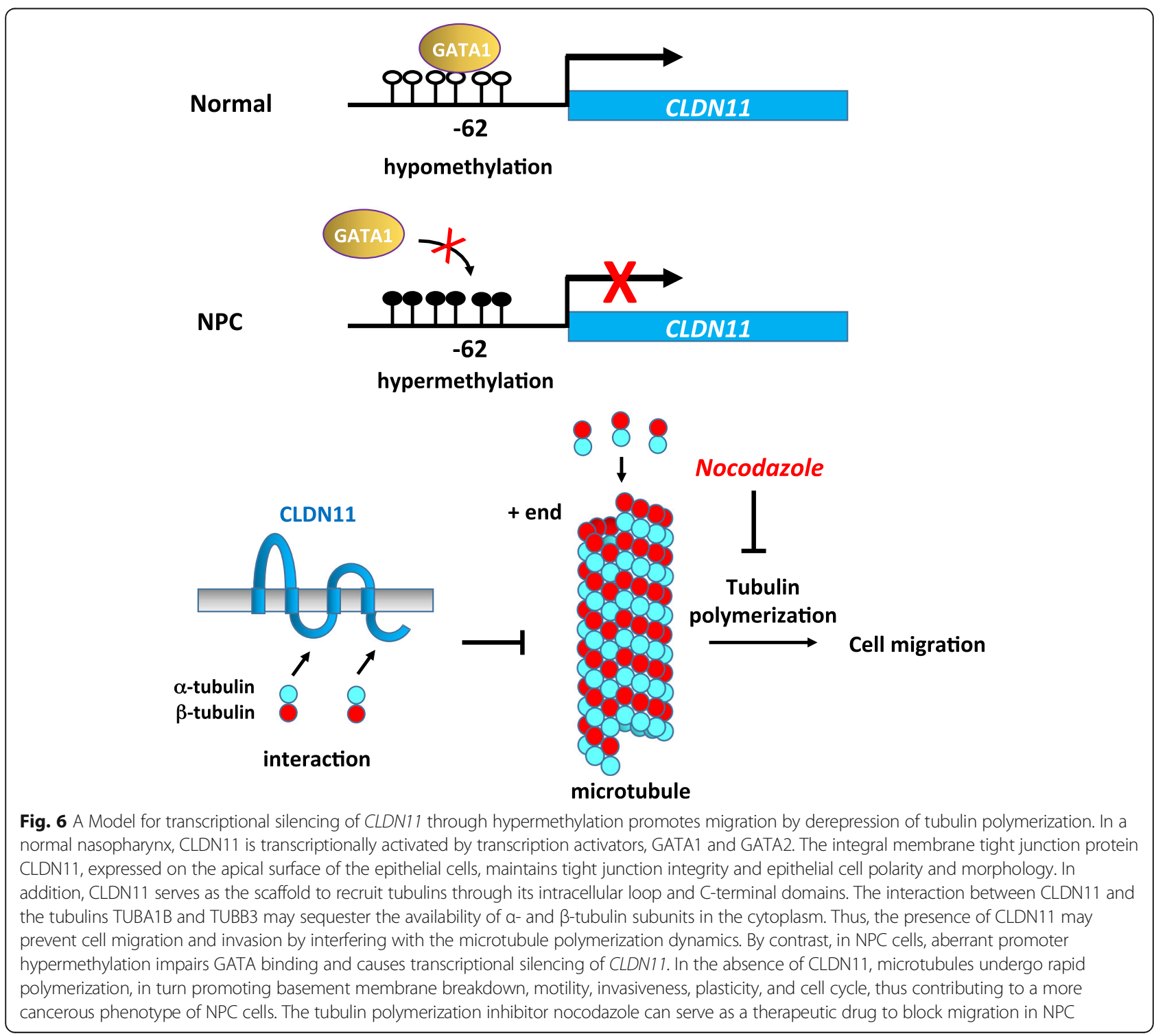


CLDN11 in NPC cells did not restore CLDN11 to its appropriate location, the plasma membrane. This mislocalized exogenous CLDN11 occurred potentially because of the downregulation of other tight junction components or defective protein trafficking mechanism in NPC cells.

Apart from being considered tight junction scaffolds, claudins may have multiple roles beyond the cell barrier including governing cell cycle, motility, transformation, and proliferation [17, 40-43]. Furthermore, different claudins may have a positive or negative influence on tumorigenesis; even the same claudin may behave differently in different cancers [44, 45]. Hence, claudins may interact with various cellular partners to execute its diverse functions. Therefore, protein-protein interaction networks determine the potential functions of claudins. In general, claudins comprise four transmembrane domains, two extracellular loops, one intracellular loop, and a short intracellular C-terminus with PDZ motif, postsynaptic density protein PSD95, Drosophila disk large tumor suppressor (Dlg1), and zonula occludens-1 protein (ZO-1) [14]. A classical claudin's PDZ motif can interact with ZOs through the last two amino acids YV at the C-terminus [46, 47]. However, unlike classical claudins, CLDN11 terminates with HV, a nonclassical PDZ domain, and it does not interact with ZOs [48]. Interestingly, the exogenous overexpression of CLDN11 reduced the migration and invasion abilities of NPC cells. Thus, in addition to its traditional roles in cell barrier, cell polarity, and paracellular transport, CLDN11 may have other cellular functions involved in cell motility regulation.

This is the first report demonstrating that microtubule components TUBA1B and TUBB3 are the two novel CLDN11 interacting proteins. Microtubules, composed of $\alpha-\beta$ tubulin heterodimers, are cytoskeleton elements involved in multiple cellular processes including mitosis, transportation, signal transduction, and motility [49, 50]. Our tubulin polymerization assay results demonstrated that CLDN11 may perturb the dynamic $\alpha-\beta$ tubulin heterodimerization. We speculate that the interaction between CLDN11 and the two tubulins may modulate microtubules remodeling, possibly influencing the cytoskeleton rearrangement, directional migratory effect, cell motility, and cell cycle in cancer cells. Furthermore, CLDN11 may provide an anchorage platform to secure the cytoskeleton microtubules and make them less flexible, thus maintaining the polarity and orderly morphology of normal epithelial cells.

Finally, we propose a working model of CLDN11 transcriptional regulation and functions in Fig. 6. Thus, CLDN11 is a bona fide tumor suppressor gene, which can be inactivated by DNA methylation. Our study provides a novel molecular mechanism underlying the anticancer function of CLDN11. Tubulin polymerization inhibitors may be used to block migration in cancer cells, which have low CLDN11 expression. Thus, CLDN11 may serve as prognostic and potential therapeutic biomarker for NPC and other cancers.

\section{Conclusions}

In this study, we identified a differentially hypermethylated and downregulated gene CLDN11 in NPC. CLDN11 exerts its migration inhibitory role through interacting with TUBA1B and TUBB3, and disturbing the tubulin polymerization. Therefore, silencing of CLDN11 increases cell plasticity and promotes NPC progression.

\section{Additional files}

Additional file 1: Supplementary Materials and Methods. (DOC 39 kb) Additional file 2: Table S1. Sequence of primers in this study. (PDF $105 \mathrm{~kb}$ ) Additional file 3: Figure S1. Differentially methylated CLDN11. Based on the methylated DNA fragment purified by methyl-binding protein affinity column, followed by next generation sequencing and DNAnexus (CA, USA) sequence analysis, CLDN11 gene region $(-723 \sim+1822)$ is enriched and differentially methylated (experimental versus background $=1.5$, C666.1 versus NP69). Schematic map of CLDN11 (- 723 + 1822) with CpG islands (blue region) is adapted from the MethPrimer website (http://www.urogene.org/methprimer/). CpG sites (orange vertical bars) and the transcription start site (+?1) are indicated. Read coverage of the methylated DNA from C666.1 and NP69 (green peaks) are visualized by DNAnexus genome browser. The orange region represents the differentially methylated peak in C666.1. (PDF 306 kb)

Additional file 4: Figure S2. Immunohistochemistry staining analysis of CLDN11 in nine paired NPC tissues with higher magnification (800X). (TIFF 8900 kb)

Additional file 5: Figure S3. Sequence of CLDN11 promoter $(-1000 \sim+200)$. The promoter sequence of CLDN11, transcription factor binding sites [GATA1(- 90), GATA1/2(- 60), GATA2(+ 184)] and transcription start sites $(+1)$ are indicated. (PDF $44 \mathrm{~kb})$

Additional file 6: Figure S4. Co-immunoprecipitation of interacting proteins of Flag-tagged CLDN11. (a) Co-immunoprecipitation assays were performed using anti-Flag M2 beads (Sigma) on cell lysates transfected with either vector ( $\mathrm{Vec}, \mathrm{V}$ ) or CLDN11-3F (C) expressing plasmid. The immunoprecipitated protein samples were separated in 12\% SDS-PAGE. The gel was stained with Coomassie Blue (left panel). (b) For Western blot analysis, the SDS-PAGE was loaded with 1\% input lysate, 25\% immunoprecipitated lysate, and $1 \%$ flow through (FT), and was detected by anti-Flag antibody. (PDF $165 \mathrm{~kb}$ )

Additional file 7: Table S2. Identified proteins from CLDN11-3flag Co-IP by LC-MS/MS. (PDF 222 kb)

Additional file 8: Identified peptide sequence of TUBA1b, TUBB3 and CLDN11-3flag by LC-MS/MS. (XLSX 19 kb)

Additional file 9: Figure S5. Tubulin polymerization assays. (a) Tubulin polymerization assays were performed in standard condition (TUB), TUB with purified GST, TUB with purified GST-CLDN11, and TUB with $10 \mu \mathrm{M}$ nocodazole (tubulin polymerization inhibitor). Tubulin polymerization was monitored using an ELISA reader (340nm) every $30 \mathrm{~s}$ for 60 min (upper panel). (b) The rate of tubulin polymerization (?mOD/min) was plotted (lower panel) and the Vmax for each reaction is indicated by an arrow. (c) The purified GST and GST-CLDN11 protein were detected by western blotting using either anti-GST or anti-CLDN11 antibody. (PDF 203 kb)

\section{Abbreviations}

5'Aza: 5'-azacytidine; ANOVA: Analysis of variance; BS: Bisulfite sequencing; ChIP: Chromatin immunoprecipitation; CLDN: Claudin; DMEM: Dulbecco's 
Modified Eagle's medium; DNMT1: DNA methyltransferase 1; EBV: EpsteinBarr virus; EMSA: Electrophoretic mobility shift assay; FBS: Fetal bovine serum; HRP: Horseradish peroxidase; LC-MS/MS: Liquid chromatography-tandem mass spectrometry; LMP1: Latent membrane protein 1; MAP: Microtubule associated protein; MBD: Methyl binding domain; NGS: Next generation sequencing; NPC: Nasopharyngeal carcinoma; OD: Optical density; PBS: Phosphate buffered saline; qRT-PCR: Quantitative real time polymerase chain reaction; RPMI: Roswell Park Memorial Institute; RT: Reverse transcription; SD: Standard deviation; TUBA1B: Tubulin alpha-1b; TUBB3: Tubulin beta-3

\section{Acknowledgements}

We thank the Proteomics Core Lab., Bioinformatics Core Lab., and Pathology Core Lab. of Molecular Medicine Research Center in Chang Gung University for technical supports. We thank Yang Ming University Genomic Center for performing NGS.

\section{Funding}

This work was supported by the grants of Hsin-Pai Li from Chang Gung Memorial Hospital, CMRPD3E0071 2, CMRPD1G0471 and BMRPB14; Ministry of Science and Technology of Republic of China (ROC), MOST-103-2320-B-182-015 (NMRPD1D0621), MOST-104-2320-B-182-033-MY3 (NMRPD1E0771 3); Ministry of Education of ROC EMRPD1E1551, EMRPD1F0171.

\section{Availability of data and materials}

Please contact author for data requests.

\section{Authors' contributions}

HPL designed research; HPL, CCP, CHC, MJS, MYH, YRL and YLC performed research; CCW performed proteomic analysis; TWC and PT performed bioinformatics analysis; YSC, KPC MD and CLH MD, contributed NPC clinical samples; HPL and CCP analyzed data and wrote paper. All authors read and approved the final manuscript.

\section{Ethics approval and consent to participate}

This study was reviewed and approved by the IRB ethics committee of CGMH (IRB:97-1226A3).

\section{Competing interests}

The authors declare that they have no competing interests.

\section{Publisher's Note}

Springer Nature remains neutral with regard to jurisdictional claims in published maps and institutional affiliations.

\section{Author details \\ 'Graduate Institute of Biomedical Sciences, Chang Gung University, No.259, Wenhua 1st Rd., Guishan Dist., Taoyuan City 333, Taiwan. ${ }^{2}$ Department of Microbiology and Immunology, Chang Gung University, No.259, Wenhua 1st Rd., Guishan Dist., Taoyuan City 333, Taiwan. ${ }^{3}$ Department of Medical Biotechnology and Laboratory Science, Chang Gung University, No.259, Wenhua 1st Rd., Guishan Dist., Taoyuan City 333, Taiwan. ${ }^{4}$ Department of Biomedical Sciences, Chang Gung University, No.259, Wenhua 1st Rd., Guishan Dist., Taoyuan City 333, Taiwan. ${ }^{5}$ Molecular Medicine Research Center, Chang Gung University, No.259, Wenhua 1st Rd., Guishan Dist., Taoyuan City 333, Taiwan. ${ }^{6}$ Bioinformatics Center, Medical School, Chang Gung University, No.259, Wenhua 1st Rd., Guishan Dist., Taoyuan City 333, Taiwan. ${ }^{7}$ Department of Otolaryngology-Head and Neck Surgery, Chang Gung Memorial Hospital, Chang Gung University, No.5, Fuxing St., Guishan Dist., Taoyuan City 333, Taiwan. ${ }^{8}$ Division of Hematology-Oncology, Chang Gung Memorial Hospital, Chang Gung University, No.5, Fuxing St., Guishan Dist., Taoyuan City 333, Taiwan.}

\section{Received: 1 March 2018 Accepted: 6 April 2018}

Published online: 10 May 2018

\section{References}

1. Baylin SB. DNA methylation and gene silencing in cancer. Nat Clin Pract Oncol. 2005;2(Suppl 1):S4-11.

2. Esteller M. Epigenetics in cancer. N Engl J Med. 2008;358:1148-59.

3. Jones PA. Overview of cancer epigenetics. Semin Hematol. 2005;42:S3-8.
4. Tsai CN, Tsai CL, Tse KP, Chang HY, Chang YS. The Epstein-Barr virus oncogene product, latent membrane protein 1, induces the downregulation of E-cadherin gene expression via activation of DNA methyltransferases. Proc Natl Acad Sci U S A. 2002;99:10084-9.

5. Tsai CL, Li HP, Lu YJ, Hsueh C, Liang Y, Chen CL, Tsao SW, Tse KP, Yu JS, Chang YS. Activation of DNA methyltransferase 1 by EBV LMP1 involves c-Jun NH (2)-terminal kinase signaling. Cancer Res. 2006;66:11668-76.

6. Jiang W, Cai R, Chen QQ. DNA methylation biomarkers for nasopharyngeal carcinoma: diagnostic and prognostic tools. Asian Pac J Cancer Prev. 2015; 16:8059-65.

7. Niller HH, Banati F, Salamon D, Minarovits J. Epigenetic alterations in Epstein-Barr virus-associated diseases. Adv Exp Med Biol. 2016;879:39-69.

8. Dai W, Zheng H, Cheung AK, Lung ML. Genetic and epigenetic landscape of nasopharyngeal carcinoma. Chin Clin Oncol. 2016;5:16.

9. Li HP, Peng CC, Chung IC, Huang MY, Huang ST, Chen CC, Chang KP, Hsu $\mathrm{CL}$, Chang YS. Aberrantly hypermethylated Homeobox A2 derepresses metalloproteinase-9 through TBP and promotes invasion in nasopharyngeal carcinoma. Oncotarget. 2013;4:2154-65.

10. Li HP, Huang HY, Lai YR, Huang JX, Chang KP, Hsueh C, Chang YS. Silencing of miRNA-148a by hypermethylation activates the integrin-mediated signaling pathway in nasopharyngeal carcinoma. Oncotarget. 2014;5:7610-24.

11. Chen LC, Chung IC, Hsueh C, Tsang NM, Chi LM, Liang Y, Chen CC, Wang $L J$, Chang YS. The antiapoptotic protein, FLIP, is regulated by heterogeneous nuclear ribonucleoprotein $\mathrm{K}$ and correlates with poor overall survival of nasopharyngeal carcinoma patients. Cell Death Differ. 2010;17: 1463-73.

12. Anderson JM, Van Itallie CM. Physiology and function of the tight junction. Cold Spring Harb Perspect Biol. 2009;1:a002584.

13. Gunzel D, Yu AS. Claudins and the modulation of tight junction permeability. Physiol Rev. 2013;93:525-69.

14. Krause G, Winkler L, Mueller SL, Haseloff RF, Piontek J, Blasig IE. Structure and function of claudins. Biochim Biophys Acta. 2008;1778:631-45.

15. Tsukita S, Furuse M. The structure and function of claudins, cell adhesion molecules at tight junctions. Ann N Y Acad Sci. 2000;915:129-35.

16. Markov AG. Claudins as tight junction proteins: the molecular element of paracellular transport. Ross Fiziol Zh Im I M Sechenova. 2013:99:175-95.

17. Runkle EA, Mu D. Tight junction proteins: from barrier to tumorigenesis. Cancer Lett. 2013:337:41-8.

18. Awsare NS, Martin TA, Haynes MD, Matthews PN, Jiang WG. Claudin-11 decreases the invasiveness of bladder cancer cells. Oncol Rep. 2011;25:1503-9.

19. Iravani O, Tay BW, Chua PJ, Yip GW, Bay BH. Claudins and gastric carcinogenesis. Exp Biol Med (Maywood). 2013;238:344-9.

20. Abe M, Yamashita S, Mori Y, Abe T, Saijo H, Hoshi K, Ushijima T, Takato T. High-risk oral leukoplakia is associated with aberrant promoter methylation of multiple genes. BMC Cancer. 2016;16:350.

21. Walesch SK, Richter AM, Helmbold P, Dammann RH. Claudin11 promoter Hypermethylation is frequent in malignant melanoma of the skin, but uncommon in nevus cell nevi. Cancers (Basel). 2015;7:1233-43.

22. Nissinen L, Siljamaki E, Riihila P, Piipponen M, Farshchian M, Kivisaari A, Kallajoki M, Raiko L, Peltonen J, Peltonen S, Kahari VM. Expression of claudin11 by tumor cells in cutaneous squamous cell carcinoma is dependent on the activity of p38delta. Exp Dermatol. 2016;6(9):7.

23. Agarwal R, Mori Y, Cheng Y, Jin Z, Olaru AV, Hamilton JP, David S, Selaru FM, Yang J, Abraham JM, et al. Silencing of claudin-11 is associated with increased invasiveness of gastric cancer cells. PLoS One. 2009:4:e8002.

24. Mimori-Kiyosue Y. Shaping microtubules into diverse patterns: molecular connections for setting up both ends. Cytoskeleton (Hoboken). 2011;68: 603-18.

25. Cheeseman IM, Desai A. Molecular architecture of the kinetochoremicrotubule interface. Nat Rev Mol Cell Biol. 2008:9:33-46.

26. Janke C, Bulinski JC. Post-translational regulation of the microtubule cytoskeleton: mechanisms and functions. Nat Rev Mol Cell Biol. 2011;12: 773-86

27. Li LC, Dahiya R. MethPrimer: designing primers for methylation PCRs. Bioinformatics. 2002;18:1427-31.

28. Zhu W, Smith JW, Huang CM. Mass spectrometry-based label-free quantitative proteomics. J Biomed Biotechnol. 2010;2010:840518.

29. Tsai HC, Baylin SB. Cancer epigenetics: linking basic biology to clinical medicine. Cell Res. 2011;21:502-17.

30. Sandoval J, Esteller M. Cancer epigenomics: beyond genomics. Curr Opin Genet Dev. 2012;22:50-5. 
31. Carmona FJ, Esteller M. DNA methylation in early neoplasia. Cancer Biomark. 2010:9:101-11.

32. Laird PW. The power and the promise of DNA methylation markers. Nat Rev Cancer. 2003:3:253-66.

33. Gao L, van den Hurk K, Moerkerk PT, Goeman JJ, Beck S, Gruis NA, van den Oord JJ, Winnepenninckx VJ, van Engeland M, van Doorn R. Promoter CpG island hypermethylation in dysplastic nevus and melanoma: CLDN11 as an epigenetic biomarker for malignancy. J Invest Dermatol. 2014;134: 2957-66.

34. Lui WY, Wong EW, Guan Y, Lee WM. Dual transcriptional control of claudin11 via an overlapping GATA/NF-Y motif: positive regulation through the interaction of GATA, NF-YA, and CREB and negative regulation through the interaction of Smad, HDAC1, and mSin3A. J Cell Physiol. 2007;211:638-48.

35. Bronstein JM, Popper P, Micevych PE, Farber DB. Isolation and characterization of a novel oligodendrocyte-specific protein. Neurol. 1996; 47:772-8.

36. Morita K, Sasaki H, Fujimoto K, Furuse M, Tsukita S. Claudin-11/OSP-based tight junctions of myelin sheaths in brain and Sertoli cells in testis. J Cell Biol. 1999;145:579-88.

37. Gow A, Southwood CM, Li JS, Pariali M, Riordan GP, Brodie SE, Danias J, Bronstein JM, Kachar B, Lazzarini RA. CNS myelin and sertoli cell tight junction strands are absent in Osp/claudin-11 null mice. Cell. 1999:99:649-59.

38. Devaux J, Gow A. Tight junctions potentiate the insulative properties of small CNS myelinated axons. J Cell Biol. 2008;183:909-21.

39. Gow A, Davies C, Southwood CM, Frolenkov G, Chrustowski M, Ng L, Yamauchi D, Marcus DC, Kachar B. Deafness in Claudin 11-null mice reveals the critical contribution of basal cell tight junctions to stria vascularis function. J Neurosci. 2004;24:7051-62.

40. Balda MS, Matter K. The tight junction protein ZO-1 and an interacting transcription factor regulate ErbB-2 expression. EMBO J. 2000;19:2024-33.

41. Singh $A B$, Sharma A, Dhawan P. Claudin family of proteins and cancer: an overview. J Oncol. 2010;2010:541957.

42. Lu Z, Kim do H, Fan J, Lu Q, Verbanac K, Ding L, Renegar R, Chen YH. A non-tight junction function of claudin-7-interaction with integrin signaling in suppressing lung cancer cell proliferation and detachment. Mol Cancer. 2015;14:120.

43. Tabaries S, Siegel PM. The role of claudins in cancer metastasis. Oncogene. 2017;36:1176-90

44. Kwon MJ. Emerging roles of claudins in human cancer. Int J Mol Sci. 2013; 14:18148-80.

45. Turksen K, Troy TC. Junctions gone bad: claudins and loss of the barrier in cancer. Biochim Biophys Acta. 1816;2011:73-9.

46. Itoh M, Furuse M, Morita K, Kubota K, Saitou M, Tsukita S. Direct binding of three tight junction-associated MAGUKs, ZO-1, ZO-2, and ZO-3, with the COOH termini of claudins. J Cell Biol. 1999;147:1351-63.

47. Van Itallie CM, Gambling TM, Carson JL, Anderson JM. Palmitoylation of claudins is required for efficient tight-junction localization. J Cell Sci. 2005; 118:1427-36.

48. Tiwari-Woodruff SK, Buznikov AG, Vu TQ, Micevych PE, Chen K, Kornblum HI, Bronstein JM. OSP/claudin-11 forms a complex with a novel member of the tetraspanin super family and beta1 integrin and regulates proliferation and migration of oligodendrocytes. J Cell Biol. 2001;153:295-305.

49. Conde C, Caceres A. Microtubule assembly, organization and dynamics in axons and dendrites. Nat Rev Neurosci. 2009;10:319-32.

50. Etienne-Manneville $\mathrm{S}$. From signaling pathways to microtubule dynamics: the key players. Curr Opin Cell Biol. 2010;22:104-11.

\section{Ready to submit your research? Choose BMC and benefit from:}

- fast, convenient online submission

- thorough peer review by experienced researchers in your field

- rapid publication on acceptance

- support for research data, including large and complex data types

- gold Open Access which fosters wider collaboration and increased citations

- maximum visibility for your research: over $100 \mathrm{M}$ website views per year

At BMC, research is always in progress.

Learn more biomedcentral.com/submissions 